\title{
A short leucocyte telomere length is associated with development of insulin resistance
}

\author{
Simon Verhulst ${ }^{1}$ - Christine Dalgård ${ }^{2}$ - Carlos Labat ${ }^{3,4}$ • Jeremy D. Kark ${ }^{5}$. \\ Masayuki Kimura $^{6}$ - Kaare Christensen ${ }^{7,8,9}$ - Simon Toupance ${ }^{3,4}$ - Abraham Aviv ${ }^{6}$. \\ Kirsten O. Kyvik ${ }^{10}$ - Athanase Benetos ${ }^{3,4,11}$
}

Received: 27 October 2015 / Accepted: 16 February 2016 / Published online: 28 March 2016

(C) Springer-Verlag Berlin Heidelberg 2016

\begin{abstract}
Aims/hypothesis A number of studies have shown that leucocyte telomere length (LTL) is inversely associated with insulin resistance and type 2 diabetes mellitus. The aim of the present longitudinal cohort study, utilising a twin design, was to assess whether shorter LTL predicts insulin resistance or is a consequence thereof.

Methods Participants were recruited between 1997 and 2000 through the population-based national Danish Twin Registry to participate in the GEMINAKAR study, a longitudinal evaluation of metabolic disorders and cardiovascular risk factors. Baseline and follow-up measurements of LTL and insulin resistance over an average of 12 years were performed in a subset of the Registry consisting of 338 (184 monozygotic and 154 dizygotic) same-sex twin pairs.

Results Age at baseline examination was $37.4 \pm 9.6$ (mean $\pm \mathrm{SD}$ ) years. Baseline insulin resistance was not associated
\end{abstract}

Electronic supplementary material The online version of this article (doi:10.1007/s00125-016-3915-6) contains peer-reviewed but unedited supplementary material, which is available to authorised users.

Athanase Benetos

a.benetos@chu-nancy.fr

1 Groningen Institute for Evolutionary Life Sciences, University of Groningen, Groningen, the Netherlands

2 Department of Public Health, Environmental Medicine, University of Southern Denmark, Odense, Denmark

3 INSERM, U1116, Vandoeuvre-les-Nancy, France

4 Université de Lorraine, Nancy, France

5 Hebrew University-Hadassah School of Public Health and Community Medicine, Jerusalem, Israel with age-dependent changes in LTL (attrition) over the follow-up period, whereas baseline LTL was associated with changes in insulin resistance during this period. The shorter the LTL at baseline, the more pronounced was the increase in insulin resistance over the follow-up period $(p<0.001)$; this effect was additive to that of BMI. The co-twin with the shorter baseline LTL displayed higher insulin resistance at follow-up than the co-twin with the longer LTL.

Conclusions/interpretation These findings suggest that individuals with short LTL are more likely to develop insulin resistance later in life. By contrast, presence of insulin resistance does not accelerate LTL attrition.

Keywords Genetics/epidemiology (all) · Human · Insulin sensitivity and resistance

6 Center of Human Development and Aging, Rutgers, The State University of New Jersey, New Jersey Medical School, Newark, NJ, USA

7 The Danish Twin Registry, University of Southern Denmark, Odense, Denmark

8 Department of Clinical Genetics, Odense University Hospital, Odense, Denmark

9 Department of Clinical Biochemistry and Pharmacology, Odense University Hospital, Odense, Denmark

10 Department of Clinical Research, University of Southern Denmark and Odense Patient data Explorative Network (OPEN), Odense University Hospital, Odense, Denmark

11 Département de Médecine Gériatrique, CHU de Nancy, 54511 Vandoeuvre-les-Nancy, France 


$\begin{array}{ll}\text { Abbreviations } & \\ \text { BL } & \text { Baseline } \\ \text { DZ } & \text { Dizygotic } \\ \text { FU } & \text { Follow-up } \\ \text { GEMINAKAR } & \text { Genes, Familiar and Common } \\ & \text { Environment for the Development of } \\ & \text { Insulin Resistance, Abdominal Adiposity, } \\ & \text { and Cardiovascular Risk Factors } \\ \text { LTL } & \text { Leucocyte telomere length } \\ \text { MZ } & \text { Monozygotic } \\ \text { TL } & \text { Telomere length }\end{array}$

\section{Introduction}

Age-dependent deregulation of nutrient sensing and telomere attrition are two key features of mammalian ageing [1]. Insulin resistance, which typically increases with age $[2,3]$, is the most common form of deregulated nutrient sensing in the general population. If both insulin resistance and telomere attrition reflect in some way the ageing process, is there a connection between telomere dynamics, i.e. telomere length (TL) and its age-dependent attrition, and insulin resistance in humans? A number of studies have addressed this question, examining associations of leucocyte TL (LTL), which largely reflects TL in other somatic cells [4], with indices of insulin resistance or with type 2 diabetes mellitus. The majority [5-12] but not all $[13,14]$ of these studies found that LTL (or TL in subsets of leukocytes) was inversely associated with insulin resistance or that LTL was shorter in patients with type 2 diabetes mellitus than in individuals without the disorder.

Conventionally, the shorter LTL in adults with the metabolic syndrome and insulin resistance has been attributed to an accelerated LTL attrition due to the associated chronic inflammatory state in these individuals. However, this concept has now been challenged by showing that LTL is largely determined early in life $[4,15]$. Thus, genetic factors [16] and the intrauterine environment [17-19] might play a role in fashioning TL, which, in turn, may have a major impact on the risk of metabolic diseases in adulthood. A recent clinical study in American Indians showed that short LTL was associated with the incidence of type 2 diabetes mellitus [20] and obesity [21]. The authors hypothesised that LTL could be used as a predictive marker of diabetes development in American Indians. Weischer et al [22] observed that increased body weight was associated with short LTL cross-sectionally, but not with LTL attrition during a 10-year period [22]. In this study, we applied a longitudinal twin design to assess the extent to which LTL dynamics predicts insulin resistance as assessed by HOMAIR and its change over a period of 12 years.

\section{Methods}

Study population At baseline, a total of 756 intact twins pairs (i.e. 1,512 twins) were recruited between 1997 and 2000 through the population-based national Danish Twin Registry to participate in the GEMINAKAR (Genes, Familiar and Common Environment for the Development of Insulin Resistance, Abdominal Adiposity, and Cardiovascular Risk Factors) study, a longitudinal evaluation of metabolic disorders and cardiovascular risk factors [23]. Individuals without a history of diabetes or cardiovascular disease underwent baseline physical examination and collection of fasting blood samples at one of two examination sites. At follow-up between 2010 and 2012, 1,435 twins were invited and 1139 (>79\%) underwent a second physical examination. Here, the twins were visited at home or at work by a mobile examination unit where a similar evaluation and blood collection were performed as at baseline. Both at baseline and at follow-up, glucose and insulin were measured in the fasting state. At baseline, skinfolds were measured three times at four different sites (biceps, triceps, subscapular and suprailiac) using a Harpenden caliper, all on the right side of the body. For this analysis, 338 same-sex twin pairs with complete follow-up were included. They consisted of 184 monozygotic (MZ) and 154 dizygotic (DZ) pairs (electronic supplementary material [ESM] Fig. 1). The study was approved by the Danish Ethics Committee (baseline, S-VF-19970271; follow-up, S-20090065) and Danish Data Protection Board (baseline, 1999-1200-441; follow-up, 2009-41-2990). All participants provided written informed consent.

Analyses and measurements Plasma glucose was measured in both visits using the hexokinase/G-6-PDH principle (Architect, Abbott, Lake Forest, IL, USA). Plasma total cholesterol, HDLcholesterol, LDL-cholesterol and triacylglycerol were analysed using enzymatic colorimetric reactions (Modular P, Roche, Rotkreuz, Switzerland). Serum insulin was analysed using a commercial time-resolved fluoroimmunoassay (Perkin-Elmer Life Sciences, Turku, Finland). Measurements of LTL were performed in duplicate on different gels by Southern blots as previously described [24]. The inter-assay coefficient of variation for the duplicate measures was $1.3 \%$. HOMA-IR was calculated according to Matthews et al [25]: HOMA-IR = (insulin $[\mathrm{pmol} / \mathrm{l}] \times 0.167) \times$ glucose $(\mathrm{mmol} / \mathrm{l}) / 22.5$.

Statistical analysis Pairwise comparisons were carried out using the Student $t$ test, Mann-Whitney and $\chi^{2}$ tests, as appropriate. Pearson correlations, Spearman-rank correlation and linear regression were used to assess associations between LTL and insulin resistance. The correlations between LTL and insulin resistance were also performed after adjusting LTL for age, sex and age, and sex and BMI. Deciles of LTL values for individuals were created to examine the individual's change in 
rank between the baseline measurement and that at follow-up. A general linear mixed model with twin identity as a random effect was used to determine the effect of LTL on insulin resistance, adjusted for age, sex, zygosity, BMI, and an interaction of sex and BMI. An interaction of zygosity with LTL was also assessed.

Continuous variables are presented as mean $\pm \mathrm{SD}$, while categorical variables are presented as percentages (\%). Insulin, glucose and insulin resistance are presented as medians and interquartile range $(25 \%, 75 \%)$.

\section{Results}

Table 1 displays the general characteristics of the participants. At baseline, there was no correlation of age or sex with insulin resistance (both $p>0.4$, tested with twin identity as a random effect in the model). By contrast, in the follow-up sample, insulin resistance tended to increase with age $\left(\mathrm{F}_{1,384.1}=3.19\right.$, $p<0.075)$ and was higher in men than women $\left(\mathrm{F}_{1,384.8}=4.03\right.$, $p<0.05)$. Twin identity explained approximately $11 \%$ of the variation in insulin resistance in these models. The contrasting results between the baseline and follow-up sample were confirmed by an examination of the change in insulin resistance from baseline to follow-up, which increased with age and was stronger in males (age: $\mathrm{F}_{1,334.2}=4.05 p<0.05$; sex: $\left.\mathrm{F}_{1,334.2}=8.07, p<0.005\right)$. BMI and fasting glucose were higher in men than women at both visits, and there was a trend for men to be older (Table 1). LTL was inversely correlated with age (slope baseline \pm SE: $-22.0 \pm 3.23$ bp/year; slope follow-up: $-2.0 \pm 3.31$; both $p<0.001$, tested with sex and twin identity in the model). LTL was shorter in men than in women in both models (baseline: $-52.7 \pm 64.6 \mathrm{bp}, \mathrm{F}_{1,327.2}=5.59, p<0.02$; follow-up $-50.5 \pm 63.6 \mathrm{bp}, \mathrm{F}_{1,326.7}=5.6, p<0.02$ ). When taking age and sex into account, BMI was negatively associated with LTL at follow-up (slope \pm SE: $-1.8 \pm 5.1 \mathrm{bp} / \mathrm{BMI}, p=0.02$ ) but not at baseline (slope \pm SE: $0.1 \pm 7.0 \mathrm{bp} / \mathrm{BMI}, p=0.98$ ). In line with these findings, individuals with long baseline LTL showed

Table 1 Mean values of age, BMI, LTL and median values of insulin, glucose and insulin resistance assessed by HOMA-IR at baseline and follow-up visits as well as LTL attrition over this period

\begin{tabular}{|c|c|c|c|c|c|c|}
\hline \multirow[t]{2}{*}{ Characteristic } & \multicolumn{2}{|l|}{ All } & \multicolumn{2}{|c|}{ Women } & \multicolumn{2}{|l|}{ Men } \\
\hline & $n$ & Mean \pm SD & $n$ & Mean \pm SD & $n$ & Mean \pm SD \\
\hline Number of participants & 676 & & 372 & & 304 & \\
\hline Birthweight (kg) & 666 & $2.62 \pm 0.51$ & 369 & $2.56 \pm 0.50$ & 297 & $2.69 \pm 0.51 * *$ \\
\hline $\mathrm{Age}_{\mathrm{BL}}$ (years) & 676 & $37.4 \pm 9.64$ & 372 & $36.8 \pm 9.47$ & 304 & $38.2 \pm 9.81$ \\
\hline $\operatorname{Age}_{\mathrm{FU}}$ (years) & 675 & $49.6 \pm 9.58$ & 372 & $49.1 \pm 9.47$ & 303 & $50.3 \pm 9.69$ \\
\hline $\mathrm{BMI}_{\mathrm{BL}}\left(\mathrm{kg} / \mathrm{m}^{2}\right)$ & 674 & $24.4 \pm 3.50$ & 370 & $23.8 \pm 3.64$ & 304 & $25.1 \pm 3.17 * *$ \\
\hline $\mathrm{BMI}_{\mathrm{FU}}\left(\mathrm{kg} / \mathrm{m}^{2}\right)$ & 675 & $25.6 \pm 4.22$ & 372 & $25.0 \pm 4.51$ & 303 & $26.4 \pm 3.71 * * *$ \\
\hline Triceps skinfold $_{\mathrm{BL}}(\mathrm{mm})$ & 480 & $8.70 \pm 1.55$ & 264 & $10.71 \pm 4.79$ & 216 & $6.24 \pm 2.63^{* * *}$ \\
\hline Biceps skinfold $_{\mathrm{BL}}(\mathrm{mm})$ & 480 & $14.91 \pm 7.54$ & 264 & $19.47 \pm 6.80$ & 216 & $9.35 \pm 3.65^{* * *}$ \\
\hline Subscapular skinfold $_{\mathrm{BL}}(\mathrm{mm})$ & 480 & $17.32 \pm 7.54$ & 264 & $18.40 \pm 7.80$ & 216 & $15.99 \pm 7.00^{* * *}$ \\
\hline Suprailiac skinfold $_{\mathrm{BL}}(\mathrm{mm})$ & 480 & $13.71 \pm 7.24$ & 264 & $14.27 \pm 7.28$ & 216 & $13.01 \pm 7.15^{*}$ \\
\hline Smoking $_{B L}(\%)$ & 676 & $29 \%$ & 372 & $30 \%$ & 304 & $29 \%$ \\
\hline Smoking $_{\mathrm{FU}}(\%)$ & 673 & $20 \%$ & 372 & $22 \%$ & 301 & $18 \%$ \\
\hline Fasting insulin $\mathrm{BL}_{\mathrm{BL}}(\mathrm{pmol} / \mathrm{l})$ & 670 & $34(24-46)$ & 369 & $34(25-46)$ & 301 & $32(23-44)$ \\
\hline Fasting insulin ${ }_{\mathrm{FU}}(\mathrm{pmol} / \mathrm{l})$ & 670 & $36(26-53)$ & 367 & $36(26-48)$ & 303 & $37(26-59)$ \\
\hline Fasting glucose $\mathrm{BL}_{\mathrm{BL}}(\mathrm{mmol} / \mathrm{l})$ & 668 & $4.7(4.4-5.0)$ & 367 & $4.6(4.4-4.9)$ & 301 & $4.8(4.5-5.1)^{* * *}$ \\
\hline Fasting glucose $\mathrm{FU}_{\mathrm{FU}}(\mathrm{mmol} / \mathrm{l})$ & 671 & $5.5(5.1-5.8)$ & 368 & $5.3(5.1-5.7)$ & 303 & $5.6(5.3-6.0)^{* * *}$ \\
\hline Insulin resistance $\mathrm{BL}_{\mathrm{BL}}$ & 666 & $1.18(0.83-1.59)$ & 366 & $1.18(0.84-1.60)$ & 300 & $1.18(0.80-1.59)$ \\
\hline Insulin resistance ${ }_{\mathrm{FU}}$ & 666 & $1.48(1.02-2.17)$ & 364 & $1.44(1.00-1.95)$ & 302 & $1.51(1.04-2.46)^{*}$ \\
\hline $\operatorname{LTL}_{\mathrm{BL}}(\mathrm{kb})$ & 640 & $6.99 \pm 0.66$ & 356 & $7.06 \pm 0.68$ & 284 & $6.89 \pm 0.63^{*}$ \\
\hline $\mathrm{LTL}_{\mathrm{FU}}(\mathrm{kb})$ & 619 & $6.75 \pm 0.64$ & 347 & $6.83 \pm 0.66$ & 272 & $6.65 \pm 0.61^{*}$ \\
\hline LTL attrition (bp/year) & 602 & $19.7 \pm 14.09$ & 339 & $19.7 \pm 14.67$ & 263 & $19.8 \pm 13.34$ \\
\hline
\end{tabular}

Data are means \pm SD or median $(25-75 \%$ interquartile range)

Results of tests for a difference between the sexes were in a model containing only sex and twin identity as a random effect, except for LTL where age was also in the model

${ }^{*} p<0.05,{ }^{* *} p<0.01, * * * p<0.001$

BL, baseline; FU, follow-up 
a smaller increase in BMI from baseline to follow-up (slope $\pm \mathrm{SE}$ : $21.4 \mathrm{bp} / \mathrm{BMI}$ change, $\mathrm{F}_{1,472.6}=6.06, p=0.01$ ). A trend for shorter LTL was observed in smokers at baseline and at follow-up but neither difference was statistically significant (baseline $-1.2 \pm 45.3 \mathrm{bp}, \mathrm{F}_{1,533.9}=0.83, p=0.4$; follow-up: $\left.-7.5 \pm 47.0 \mathrm{bp}, \mathrm{F}_{1,474.6}=2.72, p<0.1\right)$. In unadjusted analyses the skinfold values were associated with BMI and insulin resistance but not with LTL.

Previously we reported tracking and fixed ranking of LTL in adults [18]. We replicated these findings in the GEMINAKAR participants. This is displayed in three ways: first, we observed a strong correlation between LTL at baseline LTL $_{\mathrm{BL}}$ and LTL at follow-up (Fig. 1a). Second, we found that the individual's ranking of LTL (by decile) hardly changed between baseline and follow-up, as 93.7\% (95\% CI 91.5, 95.5\%) showed no change in rank or a 1 decile change over the course of the 12 years. Thus, individuals having a short or a long LTL at baseline examination showed the same at follow-up examination (Fig. 1b). Third, exploiting the twin model, we found that delta $(\Delta$; the intra-pair difference) LTL was highly correlated between baseline and follow-up examinations (Fig. 1c).

Having established tracking and fixed ranking in LTL, we next examined the association between LTL and insulin resistance. First we assessed the association between insulin resistance at baseline and LTL attrition (Fig. 2a). This analysis showed that the levels of insulin resistance did not influence LTL attrition. By contrast, changes in insulin resistance over the follow-up period were associated with LTL at baseline: the shorter the baseline LTL the more pronounced the increase in insulin resistance (Fig. 2b). Similar results were observed when fasting insulin was used in the model instead of insulin resistance. These results held after adjustment for age and sex (Table 2). Notably, insulin resistance at follow-up was associated with baseline LTL, while insulin resistance at baseline was not significantly associated with baseline LTL (Table 2). Follow-up insulin resistance was also associated with LTL at follow-up (Table 2). We included sex as a factor in these models, which explained a significant part of the variance in most cases, but interactions between sex and LTL were not significant when added to the models in Table $2(p \geq 0.1)$. Neither was there a significant effect of zygosity when added to the models in Table 2, although there was a trend for MZ twins to have higher insulin resistance at follow-up $(p=0.056$ when added to Table 2 , other $p$ values $\geq 0.14$ ). More importantly, interactions between zygosity and LTL did not explain a significant part of the variation ( $p \geq 0.28$ ). Similarly, adding age to the models in Table 2 did not change the findings (ESM Table 1).

BMI at baseline is associated with variations in insulin resistance [26], and BMI is also often associated with LTL
Fig. 1 LTL characteristics.

(a) LTL at baseline examination vs LTL at follow-up examination. (b) Ranking of individuals by deciles; 93.7\% (95\% CI 91.5\%, 95.5\%) show no rank change or a 1-decile change over time. (c) The difference in LTL between cotwins at baseline $\left(\triangle \mathrm{LTL}_{\mathrm{BL}}\right)$ and at follow-up ( $\left.\Delta \mathrm{LTL}_{\mathrm{FU}}\right)$. The cotwin with the longest LTL at baseline was considered first in the calculation of $\Delta$ for both baseline examination and followup examination. BL, baseline; FU, follow-up a
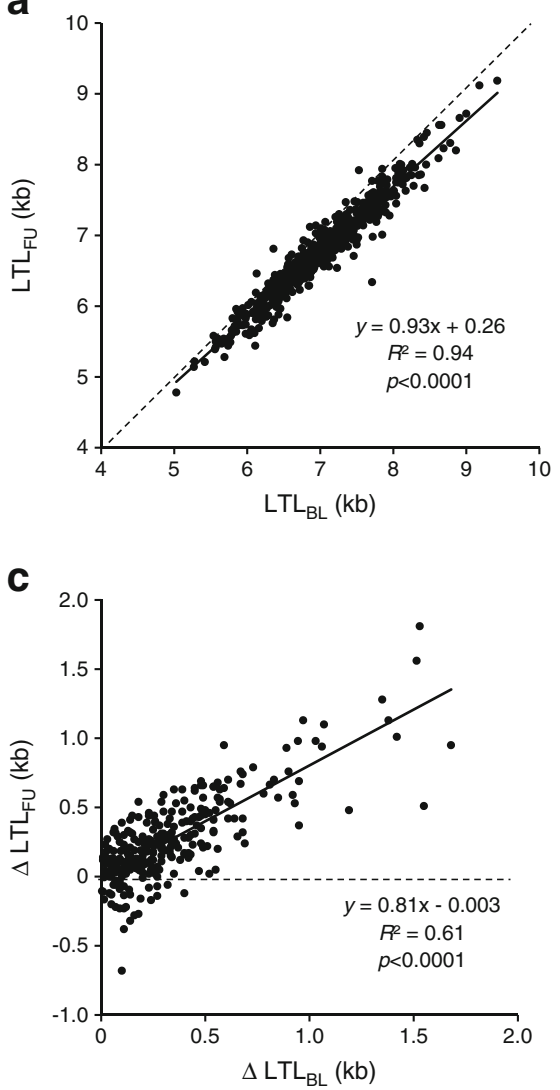

b

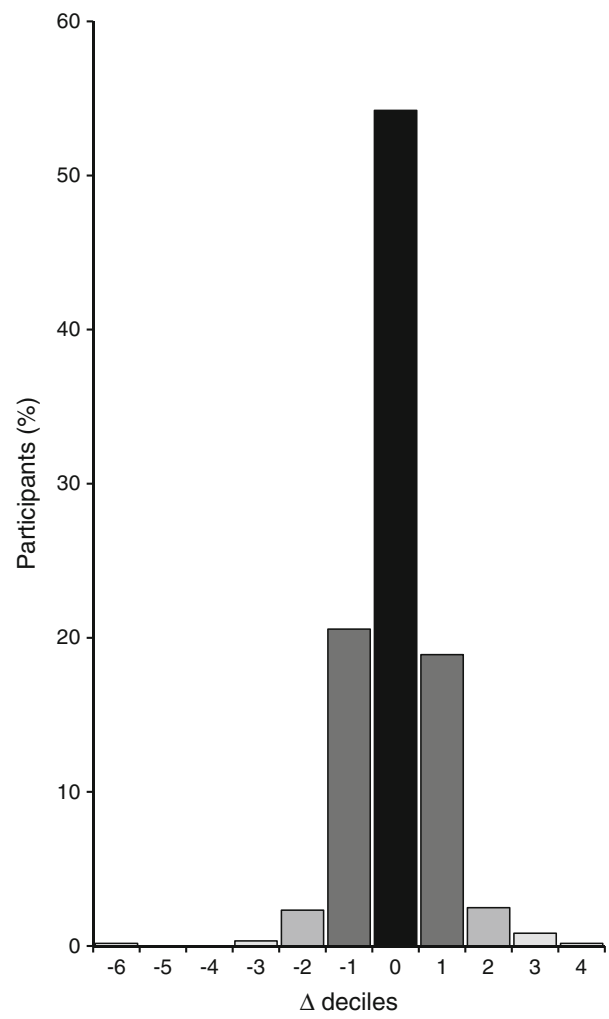



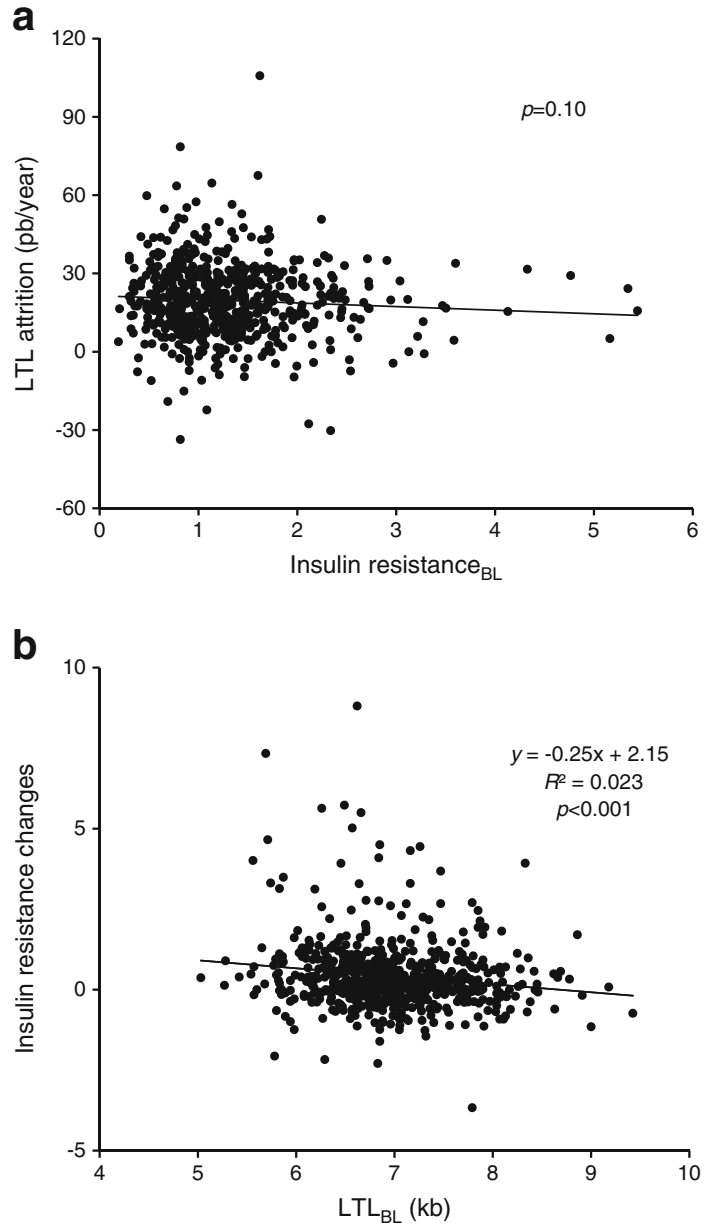

Fig. 2 Relation between LTL and insulin resistance assessed by HOMAIR. (a) The correlation between HOMA-IR at baseline and changes in LTL (LTL attrition). (b) The correlation between LTL at baseline examination and changes in HOMA-IR during the follow-up period. BL, baseline; FU, follow-up

(see example above). We therefore investigated whether LTL predicts changes in insulin resistance independent of BMI, by adding BMI to the models in ESM Table 1. The BMI effect may differ between the sexes [27, 28]; therefore, we also tested the interaction between sex and BMI in these models. A higher BMI was associated with a larger increase in insulin resistance, but adding BMI to the model in ESM Table 1 had little effect on the association between LTL at baseline and change in insulin resistance (ESM Table 2). It is noteworthy that the effect of BMI on the change in insulin resistance depended strongly on sex (interaction BMI $\times \operatorname{sex}, p=0.0004$, ESM Table 2), being negligible in women and positive in men. Adding the BMI and its interaction with sex to the models in ESM Table 2 further confirmed the result that BMI affects insulin resistance, and that this effect is additive to that of LTL.

Insulin resistance is calculated from insulin and glucose values [25], and it is of interest therefore to investigate whether the association between baseline LTL and the change in
Table 2 Insulin resistance (assessed by HOMA-IR) in relation to LTL, age and sex

\begin{tabular}{llll}
\hline Variable & \multicolumn{1}{l}{ Estimate (SE) } & $F(d f)$ & $p$ value \\
\hline \multicolumn{2}{l}{ Change in insulin resistance } & & \\
$\quad$ Intercept & $1.796(0.475)$ & - & 0.0002 \\
Sex & $0.330(0.090)$ & $13.35(1,318.2)$ & 0.0003 \\
LTL & $-0.217(0.067)$ & $10.57(1,405.1)$ & 0.001 \\
Insulin resistance at FU & & \\
$\quad$ Intercept & $3.197(0.525)$ & & $<0.001$ \\
Sex & $0.284(0.100)$ & $8.03(1,323.2)$ & 0.005 \\
LTL & $-0.251(0.074)$ & $11.65(1,428.6)$ & 0.0007 \\
Insulin resistance at BL & & $<0.001$ \\
Intercept & $1.411(0.287)$ & - & 0.5 \\
Sex & $-0.056(0.058)$ & $0.39(1,316.3)$ & 0.4 \\
LTL & $-0.038(0.040)$ & $0.87(1,495.1)$ & \\
Insulin resistance at FU & & $<0.001$ \\
Intercept & $3.063(0.527)$ & - & 0.012 \\
Sex & $0.255(0.101)$ & $6.41(1,321.3)$ & 0.0017 \\
LTL & $-0.242(0.076)$ & $9.99(1,399.2)$ & \\
\hline
\end{tabular}

Change in insulin resistance (follow-up-baseline) and LTL at baseline ( $\left.n=619, R^{2}=0.14\right)$. Insulin resistance at follow-up and LTL at baseline $\left(n=629, R^{2}=0.26\right)$

Insulin resistance and LTL at baseline $\left(n=629, R^{2}=0.56\right)$

Insulin resistance and LTL at follow-up $\left(n=611, R^{2}=0.29\right)$

All models included twin identity as a random effect, which explained 5 $39 \%$ of the variance

Sex was coded: women $=0$, men $=1$

BL, baseline; FU, follow-up

insulin resistance was due to changes in glucose, insulin or both. This question was tested by replacing the change in insulin resistance (the dependent variable) in ESM Table 2 with the change in either glucose (ESM Table 3) or insulin (ESM Table 3) level. Glucose level increased significantly from baseline to follow-up measurement (paired $t$ test, $p<0.0001$ ), but the change in glucose was not associated with baseline LTL (ESM Table 3), although the slope was negative. Insulin also increased significantly from baseline to follow-up (paired $t$ test, $p<0.0001$ ) and the change in fasting insulin level was significantly associated with baseline LTL (ESM Table 3), being larger with shorter LTL. This result suggests that the association of LTL with change in insulin resistance was mainly attributable to the change in insulin level and to a lesser extent to a change in glucose level.

Next we studied the association between insulin resistance at baseline and LTL attrition over the follow-up period. Participants with higher insulin resistance did not show more pronounced LTL attrition rates (Fig. $2 \mathrm{~b}, p=0.60$, tested with twin identity as a random effect in the model).

We then exploited the twin model to examine determinants of co-twin differences $(\Delta)$ in insulin resistance at follow-up 
visits (Table 3 and Fig. 3). These analyses were conducted in the 301 twin pairs with complete data for LTL and insulin resistance at both baseline and follow-up visits. The multivariable model (Table 3, model 2), including $\Delta$ LTL at baseline and zygosity, showed that $\Delta$ LTL was a significant determinant of $\Delta$ insulin resistance. Zygosity was not a significant determinant $(p=0.08)$. No significant interaction was observed for zygosity $\times \Delta$ LTL at baseline on insulin resistance at follow-up. Adding age and sex in the model (Table 3, model 3 ) did not modify these results. The probability that the cotwin with the shorter baseline LTL displays a higher insulin resistance at follow-up than the co-twin with the longer LTL was also tested. Wider difference of LTL between the co-twins at baseline was associated with a higher probability that the co-twin with the shorter LTL would display a higher insulin resistance than the co-twins with the longer LTL (Fig. 3). When fasting insulin was used instead of insulin resistance the results were very similar (data not shown).

\section{Discussion}

These longitudinal findings indicate that short LTL is associated with increased insulin resistance over a period of 12 years. This finding was additive to the effect of BMI; it was mainly attributable to an association of LTL with the change in insulin level and to a lesser extent to an association of LTL with the change in glucose level. In addition, insulin resistance did not increase the rate of LTL attrition over this period.

In the population at large, ageing and obesity are associated with increased insulin resistance and LTL attrition. While insulin resistance might not increase with age in all individuals [17], the evidence indicates that age-dependent LTL attrition is essentially universal in humans $[29,30]$. That being said, the effect of LTL attrition during adult life on LTL is relatively

Table 3 Logistic analysis to explain delta (between co-twins) insulin resistance at follow-up

\begin{tabular}{lcc}
\hline \multicolumn{1}{l}{ Variable } & OR $(95 \% \mathrm{CI})$ & $p$ value \\
\hline Model 1: $\Delta$ LTL $_{\mathrm{BL}}$ & $0.55(0.26,1.17)$ & 0.12 \\
Model 2: Model 1 plus zygosity $(\mathrm{MZ} \mathrm{vs} \mathrm{DZ)}$ & \\
$\quad \Delta$ LTL $_{\mathrm{BL}}$ & $0.44(0.20,0.99)$ & 0.047 \\
$\quad$ Zygosity (MZ vs DZ) & $1.53(0.94,2,49)$ & 0.08 \\
Model 3: Model 2 plus age and sex & & \\
$\quad \Delta$ LTL $_{\mathrm{BL}}$ & $0.43(0.19,0.96)$ & 0.039 \\
Zygosity (MZ vs DZ) & $1.55(0.95,2.52)$ & 0.08 \\
Age (1 year) & $1.01(0.98,1.03)$ & 0.62 \\
Sex & $1.20(0.76,1.92)$ & 0.43 \\
\hline
\end{tabular}

Analysis in 301 twin pairs (138 DZ, $163 \mathrm{MZ}$ twin pairs)

The interaction term zygosity $\times \Delta$ LTL $_{\mathrm{BL}}$ is NS $(p=0.38)$

BL, baseline

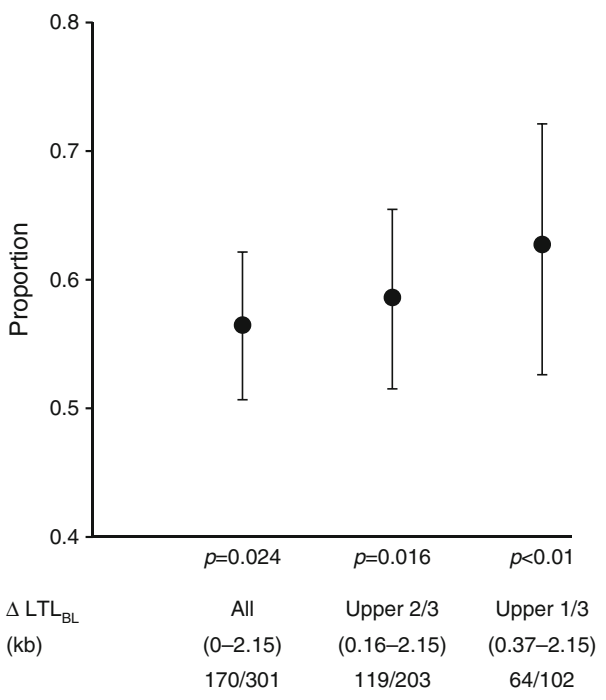

Fig. 3 Insulin resistance (assessed by HOMA-IR) according to intra-pair difference in TL among 301 same-sex twin pairs (163 MZ and $138 \mathrm{DZ}$ twin pairs). Proportion of co-twins with higher HOMA-IR and a shorter LTL ( $\Delta$ LTL) in all co-twins (all); in co-twins in the upper $2 / 3$ (wider intra-pair $\Delta$ ) LTL (upper 2/3); and in co-twins in the upper $1 / 3$ of (widest intra-pair $\Delta$ ) LTL (upper $1 / 3$ ). Numbers of twin pairs with higher HOMA-IR/total number of twins are given in the bottom line of the figure. Intra-pair $\Delta$ LTL limits are given in parentheses. Bars $=95 \% \mathrm{CI}$

small in comparison to LTL at birth and its attrition prior to adulthood. The outcome is that LTL in adults displays tracking and fixed ranking, so that individuals with a short or a long LTL at a young adult age are likely to display a correspondingly short or long LTL later in life [19]. Consequently, the overwhelming majority $(93.7 \%)$ of participants in the present study have maintained their LTL ranking as they age.

Insulin resistance is the tell-tale sign of diabetes as well as arterial ageing in general and atherosclerosis in particular [31]. The current convention is that inflammation and oxidative stress are the unifying factors that explain the associations of a relatively short LTL with atherosclerosis and with insulin resistance [16, 32]. Both atherosclerosis and insulin resistance are chronic states of indolent inflammation and oxidative stress [33]. Inflammation entails increased haematopoietic stem cell replication to accommodate the inflammatory response, a phenomenon that would heighten the rate of LTL attrition. As the $G$ triplets of the telomeres, which comprise TTAGGG repeats, are highly sensitive to the hydroxyl radical [34], at least in vitro, oxidative stress might accelerate LTL attrition due to a larger loss of telomere repeats with each replication of cells. Therefore, the shortened LTL in adults has been conventionally attributed to an accelerated LTL shortening occurring in tandem with the processes that bring about insulin resistance and atherosclerosis. In our view, this concept requires major revision, since having a long or a short LTL is principally determined prior to adulthood. One would therefore anticipate that if LTL is inversely related to insulin resistance in adults, a short LTL precedes insulin resistance. 
Recent experimental and clinical results suggest that this may be the case. Studies in rodents show that shorter telomeres may compromise beta cell function in the pancreas [35, 36], but in itself this finding is unlikely to explain the LTL-insulin resistance connection in humans, given that insulin resistance largely reflects a diminished response of peripheral tissues to insulin. In mice, short telomeres contribute to metabolic dysfunction through mitochondrial dysfunction [37], whereas mice with disruption of Rap1, a telomere-binding protein, exhibit accumulation of abdominal fat, insulin resistance and other metabolic abnormalities, suggesting a critical role of telomere biology in body weight homeostasis [38, 39].

Notably, in the present study the baseline LTL values were not correlated with baseline insulin resistance values, whereas such a relationship was found in the follow-up measurements. We suggest that these findings might relate to the young age of the cohort at the baseline visit (mean age 37.4 years), while insulin resistance in most people develops after the fourth decade. It is not surprising therefore that the association between LTL and insulin resistance appeared only in the followup visit, i.e. at a more advanced age. This supposition is supported by the positive correlation between baseline LTL and insulin resistance in the subgroup of participants older than 40 years at baseline $(p=0.025 ; n=237$, analysis as in ESM Table 1).

The findings of the present work support the idea that individuals with inherently short LTL are prone to insulin resistance because their glucose homeostasis might be impaired at a younger age than in their peers with a longer LTL. Our model does not exclude the possibility that inter-individual variation in rate of LTL attrition during adult life [28] might have some influence on the development of insulin resistance.

We would like to underline the strengths and limitations of the study. A first strength is the twin design of the study. In addition, we used the high-precision Southern blot method to measure LTL. A recent study suggested that the quantitative PCR-based method to measure LTL is as reproducible as the Southern blot method [40]. However, a commentary on the study questioned the validity of this argument [41]. In a study that impartially examined the two methods, the inter-assay coefficient of variation of the quantitative PCR method was four times as high as that of the Southern blot method [42]. We also acknowledge limitations of the study. First, participants with diabetes at the baseline visit were excluded from the study and therefore the possibility exists that diabetic patients may have an increase in the rate of LTL attrition. Second, the findings are in twins and warrant replication in singletons. However, age-adjusted values of both LTL and insulin resistance values observed in this study were very similar with those observed in singletons $[4,15,43]$.

Finally, we would like to emphasise that age-dependent deregulation of many biological networks ultimately bring about insulin resistance [44]. Thus, telomere biology might be one among many pathways that ultimately explain the rise in insulin resistance with age and the development of type 2 diabetes mellitus. Through the twin design and the sequential measurements of LTL and insulin resistance over more than a decade, the present study provides new insight into insulin resistance in humans.

Funding This work was supported by NIH grants AG030678 and HD071180; the Danish Council for Independent Research - Medical Sciences; the INTERREG 4 A - programme Southern DenmarkSchleswig-K.E.R.N. supported by the European Regional Development Fund; and the A.P. Møller Foundation for the Advancement of Medical Science. This study has been supported by the French National Research Agency (ANR), Translationnelle: N ${ }^{\circ} I D$ RCB: 2014-A00298-39: 20142017.

Duality of interest The authors declare that there is no duality of interest associated with this manuscript.

Contribution statement $\mathrm{AB}, \mathrm{AA}$ and $\mathrm{KC}$ made substantial contributions to the conception and design of the study, interpretation of data and drafting of the paper. $\mathrm{CD}, \mathrm{KOK}$ and $\mathrm{MK}$ were involved in the acquisition of the data, contributed to the interpretation of data and critically revised the paper for important intellectual content. SV contributed to the analysis and interpretation of data and drafting of the paper. JDK made a substantial contribution to conception and design of the study and critically revised the paper for important intellectual content. CL and ST contributed to the analysis and interpretation of data and critically revised the paper for important intellectual content. All authors gave final approval of the version to be published. $\mathrm{AB}$ is the guarantor of this work and, as such, had full access to all the data in the study and takes responsibility for the integrity of the data and the accuracy of the data analysis.

\section{References}

1. López-Otín C, Blasco MA, Partridge L, Serrano M, Kroemer G (2013) The hallmarks of aging. Cell 153:1194-1217

2. Ford ES, Giles WH, Dietz WH (2002) Prevalence of the metabolic syndrome among US adults: findings from the third National Health and Nutrition Examination Survey. JAMA 287:356-359

3. Morley JE (2008) Diabetes and aging: epidemiologic overview. Clin Geriatr Med 24:395-405

4. Daniali L, Benetos A, Susser E et al (2013) Telomeres shorten at equivalent rates in somatic tissues of adults. Nat Commun 4:1597

5. Gardner JP, Li S, Srinivasan SR et al (2005) Rise in insulin resistance is associated with escalated telomere attrition. Circulation 111:2171-2177

6. Demissie S, Levy D, Benjamin EJ et al (2006) Insulin resistance, oxidative stress, hypertension, and leukocyte telomere length in men from the Framingham Heart Study. Aging Cell 5:325-330

7. Aviv A, Valdes A, Gardner JP, Swaminathan R, Kimura M, Spector TD (2006) Menopause modifies the association of leukocyte telomere length with insulin resistance and inflammation. J Clin Endocrinol Metab 91:635-640

8. Al-Attas OS, Al-Daghri NM, Alokail MS et al (2010) Adiposity and insulin resistance correlate with telomere length in middle-aged Arabs: the influence of circulating adiponectin. Eur J Endocrinol 163:601-607 
9. Ahmad S, Heraclides A, Sun Q et al (2012) Telomere length in blood and skeletal muscle in relation to measures of glycaemia and insulinaemia. Diabet Med 29:e377-e381

10. Salpea KD, Talmud PJ, Cooper JA et al (2010) Association of telomere length with type 2 diabetes, oxidative stress and UCP2 gene variation. Atherosclerosis 209:42-50

11. Monickaraj F, Aravind S, Gokulakrishnan K et al (2012) Accelerated aging as evidenced by increased telomere shortening and mitochondrial DNA depletion in patients with type 2 diabetes. Mol Cell Biochem 365:343-350

12. Adaikalakoteswari A, Balasubramanyam M, Mohan V (2005) Telomere shortening occurs in Asian Indian type 2 diabetic patients. Diabet Med 22:1151-1156

13. Barbieri M, Paolisso G, Kimura M et al (2009) Higher circulating levels of IGF-1 are associated with longer leukocyte telomere length in healthy subjects. Mech Ageing Dev 130:771-776

14. Hovatta I, de Mello VD, Kananen L et al (2012) Leukocyte telomere length in the Finnish Diabetes Prevention Study. PLoS One 7: e34948

15. Benetos A, Kark JD, Susser E et al (2013) Tracking and fixed ranking of leukocyte telomere length across the adult life course. Aging Cell 12:615-621

16. Aviv A (2012) Genetics of leukocyte telomere length and its role in atherosclerosis. Mutat Res 730:68-74

17. Entringer S, Epel ES, Kumsta R et al (2011) Stress exposure in intrauterine life is associated with shorter telomere length in young adulthood. Proc Natl Acad Sci U S A 108:513-518

18. $\mathrm{Xu} \mathrm{J,} \mathrm{Ye} \mathrm{J,} \mathrm{Wu} \mathrm{Y} \mathrm{et} \mathrm{al} \mathrm{(2014)} \mathrm{Reduced} \mathrm{fetal} \mathrm{telomere} \mathrm{length} \mathrm{in}$ gestational diabetes. Plos One 9:e86161

19. Benetos A, Dalgård C, Labat C et al (2014) Sex difference in leukocyte telomere length is ablated in opposite-sex co-twins. Int J Epidemiol 43:1799-1805

20. Zhao J, Zhu Y, Lin J et al (2014) Short leukocyte telomere length predicts risk of diabetes in American Indians: the Strong Heart Family Study. Diabetes 63:354-362

21. Chen S, Yeh F, Lin J et al (2014) Short leukocyte telomere length is associated with obesity in American Indians: the Strong Heart Family Study. Aging 6:380-389

22. Weischer M, Bojesen SE, Nordestgaard BG (2014) Telomere shortening unrelated to smoking, body weight, physical activity, and alcohol intake: 4,576 general population individuals with repeat measurements 10 years apart. PLoS Genet 10:e1004191

23. Benyamin B, Sørensen TI, Schousboe K, Fenger M, Visscher PM, Kyvik KO (2007) Are there common genetic and environmental factors behind the endophenotypes associated with the metabolic syndrome? Diabetologia 50:1880-1888

24. Kimura M, Stone R, Hunt S et al (2010) Measurement of telomere length by the Southern blot analysis of the terminal restriction fragment lengths. Nat Protoc 5:1596-1607

25. Matthews DR, Hosker JP, Rudenski AS, Naylor BA, Treacher DF, Turner RC (1985) Homeostasis model assessment: insulin resistance and beta-cell function from fasting plasma glucose and insulin concentrations in man. Diabetologia 28:412-419

26. Bluher M (2014) Are metabolically healthy obese individuals really healthy? Eur J Endocrinol 171:209-219
27. Kim SH, Reaven G (2013) Sex differences in insulin resistance and cardiovascular disease risk. J Clin Endocrinol Metab 98:1716-1721

28. Shungin D, Winkler TW, Croteau-Chonka DC (2015) New genetic loci link adipose and insulin biology to body fat distribution. Nature 518:187-196

29. Steenstrup T, Hjelmborg JV, Kark JD, Christensen K, Aviv A (2013) The telomere lengthening conundrum-artifact or biology? Nucleic Acids Res 41:e131

30. Chen W, Kimura M, Kim S et al (2011) Longitudinal versus crosssectional evaluations of leukocyte telomere length dynamics: agedependent telomere shortening is the rule. J Gerontol A Biol Sci Med Sci 66:312-319

31. Avogaro A, de Kreutzenberg SV, Federici M, Fadini GP (2013) The endothelium abridges insulin resistance to premature aging. J Am Heart Assoc 2:e000262

32. Avogaro A, de Kreutzenberg SV, Fadini GP (2010) Insulin signaling and life span. Pflugers Arch 459:301-314

33. Hajjar DP, Gotto AM Jr (2013) Biological relevance of inflammation and oxidative stress in the pathogenesis of arterial diseases. Am J Pathol 182:1474-1481

34. von Zglinicki T (2012) Oxidative stress shortens telomeres. Trends Biochem Sci 27:339-344

35. Guo N, Parry EM, Li LS et al (2011) Short telomeres compromise $\beta$-cell signaling and survival. PLoS One 6:e17858

36. Tarry-Adkins JL, Chen JH, Smith NS, Jones RH, Cherif H, Ozanne SE (2009) Poor maternal nutrition followed by accelerated postnatal growth leads to telomere shortening and increased markers of cell senescence in rat islets. FASEB J 23:1521-1528

37. Sahin E, Colla S, Liesa M et al (2011) Telomere dysfunction induces metabolic and mitochondrial compromise. Nature 470:359365

38. Martinez P, Gomez-Lopez G, Garcia F et al (2013) RAP1 protects from obesity through its extratelomeric role regulating gene expression. Cell Rep 3:2059-2074

39. Yeung F, Ramirez CM, Mateos-Gomez PA et al (2013) Nontelomeric role for Rap1 in regulating metabolism and protecting against obesity. Cell Rep 3:1847-1856

40. Martin-Ruiz CM, Baird D, Roger L et al (2015) Reproducibility of telomere length assessment: an international collaborative study. Int J Epidemiol 44:1673-1683

41. Verhulst S, Susser E, Factor-Litvak PR et al (2015) Commentary: the reliability of telomere length measurements. Int J Epidemiol 44: $1683-1686$

42. Aviv A, Hunt SC, Lin J, Cao X, Kimura M, Blackburn E (2011) Impartial comparative analysis of measurement of leukocyte telomere length/DNA content by Southern blots and qPCR. Nucleic Acids Res 39:e134

43. Faerch K, Vaag A, Holst JJ, Hansen T, Jørgensen T, Borch-Johnsen K (2009) Natural history of insulin sensitivity and insulin secretion in the progression from normal glucose tolerance to impaired fasting glycemia and impaired glucose tolerance: the Inter99 study. Diabetes Care 32:439-444

44. Civelek M, Lusis AJ (2014) Systems genetics approaches to understand complex traits. Nat Rev Genet 15:34-48 